\title{
Erratum: "Structure and Expression of Gene vfr in Pseudomonas chlororaphis 449" [Russ. J. Genet., 2009, vol. 45, no. 9, p. 1055]
}

\author{
M. A. Veselova ${ }^{a}$, V. A. Lipasova, M. I. Ovadis ${ }^{b}$, L. S. Chernin ${ }^{b}$, and I. A. Khmel ${ }^{a}$ \\ ${ }^{a}$ Institute of Molecular Genetics, Russian Academy of Sciences, Moscow, 123182 Russia; \\ e-mail: khmel@img.ras.ru \\ ${ }^{b}$ Otto Warburg Center for Biotechnology, Hebrew University of Jerusalem, Rehovot 76100, Israel \\ Received January 11, 2009
}

DOI: $10.1134 / \mathrm{S} 1022795410020183$

In the abstract to the paper by M.A. Veselova et al. "Structure and Expression of Gene vfr in Pseudomonas chlororaphis 449" (Russ. J. Genet., 2009, vol. 45, no. 9, p. 1055), the sentence "The cloned vfr gene of P. chlororaphis 449 was complemented partially the mutation at gene $c r p$ in cells......" should read "The cloned $v f r$ gene of $P$. chlororaphis 449 partially complemented the mutation at gene $c r p$ in cells 